\title{
Forensic Pathological Analysis of Death Due to Pulmonary Thromboembolism: a Retrospective Study Based on 145 Cases
}

\section{Ziyuan Chen}

China Medical University

\section{Pengfei Wang}

China Medical University

\section{Mengzhou Zhang}

China University of Political Science and Law

\section{Shuheng Wen}

China Medical University

\section{Hao Cheng}

China Medical University

\section{Ning Wang}

China Medical University

\section{Mingzhe Wu}

China Medical University

\section{Bingxuan Li}

Criminal Investigation Police University of China

\section{Xiangshen Guo}

China Medical University

\section{Yujian Zhang}

China Medical University

\section{Dawei Guan}

China Medical University

\section{Linlin Wang}

China Medical University

Rui Zhao ( $\nabla$ rhao@cmu.edu.cn )

China Medical University

\section{Research Article}

Keywords: Forensic pathology, Pulmonary thromboembolism, Deep vein thrombosis, Autopsy, Sudden unexpected death 
Posted Date: February 25th, 2021

DOI: https://doi.org/10.21203/rs.3.rs-244842/v1

License: (c) (i) This work is licensed under a Creative Commons Attribution 4.0 International License. Read Full License 


\section{Abstract}

Pulmonary thromboembolism (PTE) is a common cause of sudden unexpected death in forensic practice following deep vein thrombosis (DVT). It remains easy to overlook the special procedure used for the detection of PTE during autopsies; therefore, the relationship between PTE and the associated risk factors is in need of analysis. In the present study, 145 fatal cases of PTE found during autopsies performed from 2004 to 2019 at the Center of Forensic Investigation of China Medical University were retrospectively evaluated; the demographic data, risk factors, original location of DVT, and time interval from the formation of DVT to PTE were analyzed. In addition, the difference in lung-to-heart weight ratio between the PTE and disease-free accident groups was calculated with matching for gender and age. The 40-59 age group accounted for more than half of the total cases (51.03\%). Immobilization, trauma or fracture (especially of the pelvis, femur, tibia, or fibula), surgery, pregnancy and cesarean section, mental disorders and the use of antipsychotics were the top 5 high-risk factors for fatal PTE. Among the victims, 92.9\% (130/140) died within 60 days of the first exposure to risk factors. Most DVT were formed and shed in lower limb veins, especially popliteal veins and their branches, which caused $87.6 \%$ of the thrombi distributed in bilateral pulmonary arteries. No significant difference in the lung-to-heart weight ratio was found between the PTE and control groups. The present study provides valuable information for the prevention and treatment of thrombosis during clinical events and may also be important for alerting forensic examiners to conduct special PTE detection in cases with potential risk factors.

\section{Introduction}

Pulmonary thromboembolism (PTE), which refers to pulmonary circulation disorder and cardiac dysfunction caused by deep vein thrombosis (DVT) occluding blood vessels of the pulmonary arterial tree[1], is the third leading cause of death from cardiovascular disease[2]. Epidemiological studies show that the mortality rate in untreated PTE patients is as high as $30-35 \%$, and more than $10 \%$ of inpatient deaths are related to pulmonary embolism[3, 4]. Although our understanding of the risk factors and pathogenesis of DVT has increased over recent decades, it remains an important medical issue worldwide due to its high mortality rate in both clinical and forensic practice.

DVT, a complex multifactorial disease with severe consequences, involves the interaction between acquired or inherited predispositions for thrombosis and various risk factors[5]. The acquired risk factors, which are considered the main risk factors for PTE, include injury, fracture, surgery and extended hospital admission, cancer, inflammatory disorders, infection, and use of female hormones[6]. These conditions trigger one or more risk factors for DVT by inducing hypercoagulability, stasis, or vascular wall damage/dysfunction[7]. Approximately $70 \%$ of patients with PTE have venous thrombosis of the lower extremities and thrombosis in the veins of the upper limbs or heart[7]. The high morbidity and mortality rates of DVT not only pose challenges related to clinical prevention and treatment but also to the determination of forensic causes of death and the evaluation of medical disputes. Thus, a comprehensive analysis of a large number of PTE cases would be instrumental in clinical prevention as well as court proceedings. 
To analyze the relationship between PTE and potential risk factors, 145 cases of PTE were collected and analyzed based on demographic data, potential risk factors, and anatomical and pathological examination. We found that immobilization, trauma or fracture, surgery, pregnancy and cesarean section, mental disorders and use of antipsychotics were the top 5 high-risk factors for fatal PTE. We provide valuable information regarding demographic distribution and the potential risk factors for PTE, which offers an important reference for the clinical prevention and treatment of PTE.

\section{Materials And Methods}

\section{Source of data}

A total of 145 autopsy cases of PTE were screened and collected at the Center of Forensic Investigation of China Medical University from 2005 to 2019. An additional 52 disease-free, gender- and age-matched cases of instant death due to traffic accidents were selected as the control group. All cases were confirmed by thorough autopsy, histopathological examination, toxicological detection, clinical history, and scene investigation reports. Details of the demographics, clinical diagnosis and treatment, autopsy records, histopathological examination, and toxicological screens were collected and further analyzed.

\section{Methods}

The following information was collected from expert death reports and further analyzed: demographics information, medical and medication history, type of injury, mental state, any other high-risk factors, time interval from the occurrence of the potential risk factor to the PTE, and characteristics of the thrombosis. In addition, the lung-to-heart weight ratio was compared between the PTE and disease-free accident groups.

\section{Statistical analysis}

Data were analyzed using GraphPad Prism version 8.0.0 for Windows (GraphPad Software, San Diego, California USA). Quantitative data are expressed as the mean \pm standard deviation. Categorical data are expressed as counts and percentages. The Chi-square test and unpaired $t$-test were used to analyze the differences between the two groups. Two-tailed $P$ values $₫ 0.05$ were considered significant.

\section{Ethical statement}

Procedures performed in studies involving human participants were in accordance with the ethical standards of the Medical Ethics Committee of China Medical University. Informed consent was obtained from all participants' guardians.

\section{Results}

\section{Demographics analysis of PTE cases}


A total of 145 cases were confirmed as PTE by autopsy and histopathological examination. The age ranged from 17 to 88 years, including 76 males and 69 females. The mean age of the victims was 51.5 years, with a mean age of 54.4 years for males and 48.4 years for females. Victims over 40 years old accounted for the majority $(113 / 145,77.9 \%)$ suffering from PTE (Table 1$)$. There were more men $(68 / 113$, $60.18 \%)$ than women $(45 / 113,39.82 \%)$ in the over 40 age groups (Tables 1 and 2$)$. In contrast, women accounted for the majority of victims under the age of 40 (14).

\section{Risk factors}

We analyzed the cases based on the main risk factors listed in Table 2. The number of PTE for each risk factor was counted and it was found that hospitalization or immobilization, trauma or fracture, surgery, pregnancy and cesarean section, and mental disorders and the use of antipsychotics were the top five high-risk factors, accounting for $81.4 \%, 59.3 \%, 9.7 \%, 9.7 \%$, and $6.2 \%$ of the cases, respectively. Moreover, cancer $(2.1 \%)$, diabetes $(1.4 \%)$, electrical injury $(0.7 \%)$, and no obvious risk factors $(3.4 \%)$ were also associated with some cases. For cases with the risk factor of pregnancy and cesarean section, the mean age was $29.1 \pm 5.0$ (range 21-35) years. Although we calculated the frequency of occurrence of the potential risk factors separately, the victims with PTE often possessed multiple risk factors. The number of cases with overlapping risk factors were also counted and are shown in Table 2.

\section{Time interval from the occurrence of the main risk factors to PTE}

The interval from the occurrence of these potential risk factors to death was calculated for all 140 cases. The time interval from the occurrence of the primary risk factors to the PTE varied from less than one day to several months, with a peak at 8-15 days. A total of 130 (92.9\%) victims developed PTE within 60 days following the occurrence of the main risk factors, suggesting a dangerous window for PTE (Figure 1a). We also analyzed cases with different risk factors separately. The immobilization or bedridden and trauma or fracture cases are listed in Figure $1 \mathrm{~b}-\mathrm{d}$. For surgery cases, all 14 occurred within 15 days, peaking at 2-7 days $(10 / 14,71.4 \%)$ (Figure 1d). For cesarean section cases, all 14 victims died within 15 days of cesarean section (Figure 1e). Regarding mental disorders and the use of antipsychotics, 5 of the 9 victims who suffered PTE had a history of antipsychotics use longer than 180 days (Figure 1f).

\section{Size, origin, and embolism siteof the thrombi}

The sources of the thrombi and the embolism site in cases involving different risk factors are shown in Table 3. According to the forensic autopsy data, thrombi from the veins of the lower limbs, which were mainly distributed in the femoral veins and the posterior tibial veins and their branches, accounted for $65.5 \%$ of all cases. Most of the thrombi were from the lower limbs and blocked the bilateral pulmonary arteries $(87.6 \%)$ (length, $22.7 \pm 15.4 \mathrm{~cm}$; diameter, $1.12 \pm 0.37 \mathrm{~cm}$ ). The second main source of thrombi that blocked the pulmonary arteries was the pelvic veins (length, $13.92 \pm 5.84 \mathrm{~cm}$; diameter, $1.3 \pm 0.27$ $\mathrm{cm}$ ), followed by the inferior vena cava (length, $14.83 \pm 3.01 \mathrm{~cm}$; diameter $1.28 \pm 0.27 \mathrm{~cm}$ ). In 5 cases, newly formed thrombi were found in the branches of the pulmonary artery, which were also distributed 
widely in multiple veins. In many cases, no thrombi were detected in the lower limbs (Table 3) but most had potential risk factors.

\section{The lung-to-heart weight ratio}

In forensic practice, ischemia in the anterior margin of the lung is a valuable phenomenon for the diagnosis of PTE during autopsy. In the present study, the lung-to-heart weight ratio was calculated in 110 PTE victims and 52 age- and gender-matched accident victims. Unfortunately, there was no significant difference between the two groups (Table 3); however, the weight of the heart in the PTE group was higher than that in the control group (data not shown).

\section{Discussion}

PTE, one of the leading causes of sudden death, is common clinically since it lacks specific symptoms and is frequently accompanied by trauma and/or disease. Therefore, PTE not only poses challenges in clinical prevention and treatment but also puts forward higher requirements for forensic pathologists in identifying the causes of death. Here, we analyzed the characteristics of the population, risk factors, latency, source of thrombosis, distribution of thrombi in the pulmonary arteries, and lung-to-heart weight ratio for the forensic pathologist to determine the cause of death and analyze the relationship between risk factors and PTE, in addition to providing a critical window for the prevention of PTE.

PTE is a complex multifactorial disease for which many genetic and acquired risk factors have been identified $[1,2,6,8,9]$. Among the main risk factors, surgery and extended hospital admission are considered closely related. Various diseases, including cancer, inflammatory disorders, and infection, are deemed to be responsible for the formation of thrombi. In addition, the use of female hormones, pregnancy, and puerperium increase the risk of thrombogenesis[1, 2, 6]. Traditionally, blood stasis, hypercoagulable state, and vascular endothelial damage are considered the main reasons for thrombosis[3]. In the present study, trauma and fracture caused by traffic accidents or falls were the most frequent types of injuries that induced PTE, among which, fractures of the pelvis, femur, tibia, and fibula were most often observed (72.7\%). Fractures in these areas undoubtedly lead to immobilization or being bedridden and possibly require surgical intervention. On one hand, coagulation factors are activated due to bleeding and extensive soft tissue damage, and inflammatory reactions lead to increased blood viscosity [ref]. On the other hand, prolonged immobilization causes blood stasis in the venous system of the lower limbs[6]. Moreover, high-risk factors, such as pregnancy and cesarean section (9.7\%), use of antipsychotics $(6.2 \%)$, and cancer (2.1\%), were discovered in our study, in addition to other diseases and electrical injuries also showing a correlation with PTE (Table 2); however, these were not leading causes. Previous studies have provided evidence of a link between PTE, rheumatoid arthritis, and longterm air travel[10,11]; however, these risk factors were not involved in our study. Taken together, a detailed understanding of the case by forensic pathologists and selection of potential high-risk factors will help to reduce the probability of missing PTE, in addition to analyzing the causal relationship between risk factors and death. 
It is estimated that the incidence of PTE is approximately 100-200 cases per 100,000 individuals, and the risk does not differ according to gender; although, it appears to be higher in men than in women when venous thromboembolism related to pregnancy and estrogen therapy are not considered[2, 6]. In the present study, there were slightly more male than female cases (Table 1), which is in accordance with previous reports. However, due to gender-dependent factors such as pregnancy and cesarean section, the number of female cases was higher than that of male cases under the age of $40(P<0.05)$ (Table 1), and PTE following cesarean section accounted for $58.3 \%$ of female victims in this age group. This agedependent risk factor appears to be the detrimental factor for individuals in an age group with a relatively low risk of PTE (age <40) (Table 1). Many factors are involved in the pathogenesis of PTE, including an enlarged uterus and shift in global hemostatic valance to a hypercoagulable state during pregnancy, vascular endothelial injury, and postoperative bed rest during or after cesarean section[12, 13]. Moreover, all victims suffered PTE within a critical period of 15 days of cesarean section and, based on the autopsy findings, the thrombi mainly originated from the pelvic and femoral veins. Our results suggest that 15 days after cesarean section is an essential period for PTE. In addition to the lower limb veins, the pelvic vein is an important source of thrombi that requires detailed examination, and it is suggested that the pelvic vein should be inspected during forensic autopsy under the circumstances of cesarean section.

Previous studies have reported a potential link between mental illness, use of antipsychotic drugs, and PTE[10, 11, 14-16]. In the present study, victims with mental illness or antipsychotic drug use appear to form an independent group. As shown in Table 2, we found 9 victims: 7 females and 2 males aged 47.3 \pm 7.9 years. All patients had been diagnosed with mental disorders and used antipsychotic drugs, such as clozapine, olanzapine, and chlorpromazine, for a certain period. Dima et al. retrospectively analyzed 23 reported cases of clozapine-related PTE and found that $87 \%$ occurred within six months[14]. In our series, as shown in Figure 1, 4 patients had taken antipsychotic drugs for less than six months and the other five for 3 to more than 20 years; however, given the limitation of sample size in our study, the relationship between the length of time taking antipsychotic drugs and PTE may not be accurately reflected. Nevertheless, the relationship between the use of antipsychotic drugs and DVT has been continually revealed, with the possible reasons being attributed to the fact that: (1) the sedative effect of antipsychotic drugs reduces the amount of exercise in patients with mental illness, thereby increasing venous blood siltation[16]; and (2) clozapine and olanzapine can cause obesity, which is associated with both venous stasis and hypercoagulability by decreasing fibrinolytic activity, enhancing platelet activation, and increasing levels of coagulation factors and fibrinogen[10, 14, 17]. In fact, DVT has been reported to be a psychological disease in itself, and it has even been highlighted that the effect of antipsychotic treatment may not be the only factor leading to venous thromboembolism but psychosis itself may play a certain role, which is supported by evidence that higher levels of p-selectin and Factor VIII can be detected in mental patients[15]. It is noteworthy that restriction of patient posture during the onset of mental illness appeared in some of our cases, which is an essential factor leading to venous blood stasis and increasing the incidence of PTE. According to our autopsy findings, the pulmonary arteries and venous system (especially the iliac vein, femoral vein, and posterior tibial vein) should be paid close attention for the presence of thrombi in such cases. 
Not all PTE are lethal due to the dual blood supply to the lung. The thrombi that cause fatal PTE are often widely distributed and obstruct at least two or more lobe arteries, which is in accordance with our findings that $87.6 \%$ of cases had thrombi distributed in bilateral pulmonary arteries. During autopsy, whitening of the leading edge of the lung, parenchymal ischemia and contraction, enlargement of the right heart auricle, and right heart congestion can be observed[18]. However, our results show no significant difference between the PTE and control groups with respect to the lung-to-heart weight ratio (Figure 2); although the weight of the heart in the PTE group was higher than that in the control group (data not shown), which is consistent with the post-mortem cardiac findings in PTE patients reported by Aysun Yakar et al.[19]. During forensic examination, in addition to checking the venous system of the lower extremities to elucidate the origin of thrombi, the veins adjacent to the injured site are also essential origins of thrombi and should not be overlooked.

\section{Conclusion}

In summary, we confirmed multiple high-risk factors by analyzing the pre-mortem data and autopsy results of 145 PTE cases. Moreover, we found that the key incubation period for PTE is less than 60 days after the emergence of risk factors. These results indicate that clinical prevention and treatment should focus on this critical period. Forensic pathologists should screen out relevant risk factors and be vigilant regarding the occurrence of PTE in cases possessing such risk factors, in addition to conducting a special examination of the pulmonary arteries for PTE and the possible original vessels of the thrombus.

\section{Declarations}

\section{Author contributions statement}

Linlin Wang, Rui Zhao, Dawei Guan: conceived the study. Ziyuan Chen, Pengfei Wang, Rui Zhao: collect and analyzed data, wrote the manuscript. Mengzhou Zhang, Shuheng Wen, Hao Cheng, Ning Wang, Mingzhe Wu, Bingxuan Li, Xiangshen Guo, Yujian Zhang: collected data.

\section{Additional Information}

No conflict of interest exits in the submission of this manuscript.

\section{References}

1. Margiotta G, Coletti A, Severini S, Tommolini F, Lancia M. Medico-Legal Aspects of Pulmonary Thromboembolism. Adv Exp Med Biol. 2017;906:407-18. doi:10.1007/5584_2016_130.

2. Giordano NJ, Jansson PS, Young MN, Hagan KA, Kabrhel C. Epidemiology, Pathophysiology, Stratification, and Natural History of Pulmonary Embolism. Tech Vasc Interv Radiol. 2017;20(3):13540. doi:10.1053/j.tvir.2017.07.002. 
3. Dalen JE. Pulmonary embolism: What have we learned since Virchow? - Natural history, pathophysiology, and diagnosis. Chest. 2002;122(4):1440-56. doi:DOI 10.1378/chest.122.4.1440.

4. Wells PS, Ihaddadene R, Reilly A, Forgie MA. Diagnosis of Venous Thromboembolism: 20 Years of Progress. Ann Intern Med. 2018;168(2):131-40. doi:10.7326/m17-0291.

5. Yakar A, Yakar F, Ziyade N, Yildiz M, Uzun I. Fatal pulmonary thromboembolism. Eur Rev Med Pharmacol Sci. 2016;20(7):1323-6.

6. Huisman MV, Barco S, Cannegieter SC, Le Gal G, Konstantinides SV, Reitsma PH et al. Pulmonary embolism. Nat Rev Dis Primers. 2018;4:18028. doi:10.1038/nrdp.2018.28.

7. Goldhaber SZ, Bounameaux H. Pulmonary embolism and deep vein thrombosis. Lancet. 2012;379(9828):1835-46. doi:10.1016/s0140-6736(11)61904-1.

8. Comment L, Varlet V, Ducrot K, Grabherr S. A fatal case of oxygen embolism in a hospital. Forensic Sci Res. 2017;2(2):100-6. doi:10.1080/20961790.2017.1329695.

9. Ali Z, Bolster F, Goldberg E, Fowler D, Li L. Systemic air embolism complicating upper gastrointestinal endoscopy: a case report with post-mortem CT scan findings and review of literature. Forensic Sci Res. 2016;1(1):52-7. doi:10.1080/20961790.2016.1252898.

10. Hagg S, Jonsson AK, Spigset O. Risk of venous thromboembolism due to antipsychotic drug therapy. Expert Opin Drug Saf. 2009;8(5):537-47. doi:10.1517/14740330903117271.

11. Heit JA. The epidemiology of venous thromboembolism in the community. Arterioscler Thromb Vasc Biol. 2008;28(3):370-2. doi:10.1161/ATVBAHA.108.162545.

12. Bain E, Wilson A, Tooher R, Gates S, Davis LJ, Middleton P. Prophylaxis for venous thromboembolic disease in pregnancy and the early postnatal period. Cochrane Database Syst Rev. 2014(2):CD001689. doi:10.1002/14651858.CD001689.pub3.

13. Kovacevich GJ, Gaich SA, Lavin JP, Hopkins MP, Crane SS, Stewart J et al. The prevalence of thromboembolic events among women with extended bed rest prescribed as part of the treatment for premature labor or preterm premature rupture of membranes. American Journal of Obstetrics and Gynecology. 2000;182(5):1089-92. doi:DOI 10.1067/mob.2000.105405.

14. Sarvaiya N, Lapitskaya Y, Dima L, Manu P. Clozapine-Associated Pulmonary Embolism: A HighMortality, Dose-Independent and Early-Onset Adverse Effect. Am J Ther. 2018;25(4):e434-e8. doi:10.1097/mjt.0000000000000806.

15. Hagg S, Spigset O. Antipsychotic-induced venous thromboembolism: a review of the evidence. Cns Drugs. 2002;16(11):765-76. doi:10.2165/00023210-200216110-00006.

16. Wagstaff AJ, Bryson HM. Clozapine. Cns Drugs. 1995;4(5):370-400. doi:10.2165/00023210199504050-00006.

17. Axelsson S, Hagg S, Eriksson AC, Lindahl TL, Whiss PA. In vitro effects of antipsychotics on human platelet adhesion and aggregation and plasma coagulation. Clin Exp Pharmacol Physiol. 2007;34(8):775-80. doi:10.1111/j.1440-1681.2007.04650.x. 
18. Cao Z, He ST, Zhu BL. Forensic investigation of pulmonary thromboembolism. Chinese journal of forensic medicine. 2012;27(4):32-3+7.

19. Yakar A, Yakar F, Ziyade N, Yıldız M, Üzün İ. Cardiac Findings of Pulmonary Thromboembolism by Autopsy: A Review of 48 Cases. Med Sci Monit. 2016;22:1410-4. doi:10.12659/msm.897695.

\section{Tables}

Table 1

The age and gender distributions of the enrolled population, ${ }^{*} p<0.05$

\begin{tabular}{|llllll|}
\hline Age (years) & Male $(\mathrm{n}=76)$ & Female $(\mathrm{n}=69)$ & Total & $\%$ & P-value \\
\hline$<20$ & 0 & 1 & 1 & 0.69 & \\
\hline $20-39$ & 8 & 23 & 31 & 21.38 & $0.0004^{\star}$ \\
\hline $40-59$ & 43 & 31 & 74 & 51.03 & \\
\hline 26 & 25 & 14 & 39 & 26.90 & \\
\hline
\end{tabular}

Table 2

Characteristics of involved risk factors

\begin{tabular}{|lllll|}
\hline High-risk factor & $\begin{array}{l}\text { Number of } \\
\text { cases (\%) }\end{array}$ & $\begin{array}{l}\text { Cases containing } \\
\text { overlapping risks }\end{array}$ & $\begin{array}{l}\text { Mean age } \\
\text { (SD) }\end{array}$ & M/F \\
\hline Immobilization or bedridden & $118(81.4)$ & 113 & $\begin{array}{l}52.9 \\
(14.7)\end{array}$ & $69 / 49$ \\
\hline Trauma or fracture & $86(59.3)$ & 85 & $\begin{array}{l}57.0 \\
(13.0)\end{array}$ & $59 / 27$ \\
\hline Surgery & $14(9.7)$ & 13 & $\begin{array}{l}51.8 \\
(16.0)\end{array}$ & $7 / 7$ \\
Pregnancy and cesarean & $14(9.7)$ & 9 & $29.1(5.0)$ & $0 / 14$ \\
\hline $\begin{array}{l}\text { Mental disorders and use of } \\
\text { antipsychotics }\end{array}$ & $9(6.2)$ & 3 & $47.3(7.9)$ & $2 / 7$ \\
\hline Cancer & $3(2.1)$ & 3 & $\begin{array}{l}51.0 \\
(12.5)\end{array}$ & $2 / 1$ \\
\hline Diabetes & $2(1.4)$ & 1 & 40.0 & $1 / 1$ \\
\hline Electrical injury & $1(0.7)$ & 0 & $(18.4)$ & \\
\hline No obvious risk factors & $5(3.4)$ & - & 46.0 & $1 / 0$ \\
\hline
\end{tabular}


Table 3

The origin and embolism location of thrombi corresponding to a single risk factor. $\mathrm{BP}=$ bilateral pulmonary artery, LP = left pulmonary artery, RP = right pulmonary artery

\begin{tabular}{|c|c|c|c|c|c|c|c|c|}
\hline \multirow[t]{2}{*}{ Risk factor } & \multirow{2}{*}{$\begin{array}{l}\text { Number } \\
\text { of cases }\end{array}$} & \multicolumn{4}{|c|}{ Thrombus origin } & \multicolumn{3}{|c|}{ Embolism site } \\
\hline & & $\begin{array}{l}\text { Lower } \\
\text { limb } \\
\text { veins }\end{array}$ & $\begin{array}{l}\text { Inferior } \\
\text { vena } \\
\text { cava }\end{array}$ & $\begin{array}{l}\text { Pelvic } \\
\text { veins }\end{array}$ & Undetected & BP & LP & RP \\
\hline $\begin{array}{l}\text { Immobilization or } \\
\text { bedridden }\end{array}$ & 118 & 81 & 3 & 9 & 30 & 102 & 9 & 7 \\
\hline Trauma or facture & 89 & 55 & 1 & 7 & 28 & 73 & 9 & 7 \\
\hline Surgery & 14 & 9 & 0 & 1 & 4 & 13 & 0 & 1 \\
\hline $\begin{array}{l}\text { Pregnancy and } \\
\text { cesarean section }\end{array}$ & 14 & 5 & 0 & 4 & 5 & 13 & 1 & 0 \\
\hline $\begin{array}{l}\text { Mental disorders and } \\
\text { use of } \\
\text { antipsychotics }\end{array}$ & 9 & 7 & 0 & 0 & 2 & 8 & 1 & 0 \\
\hline Cancer & 3 & 1 & 0 & 0 & 2 & 2 & 1 & 0 \\
\hline Diabetes & 2 & 1 & 0 & 0 & 1 & 1 & 0 & 1 \\
\hline Electrical injury & 1 & 0 & 0 & 0 & 1 & 1 & 0 & 0 \\
\hline $\begin{array}{l}\text { No obvious risk } \\
\text { factors }\end{array}$ & 5 & 4 & 0 & 0 & 1 & 4 & 0 & 1 \\
\hline
\end{tabular}

Figures 
(a)

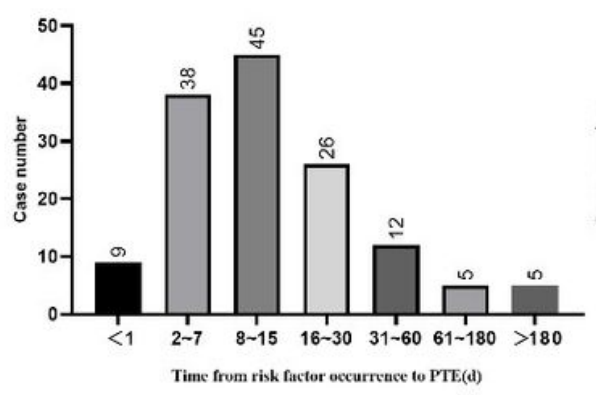

(d)

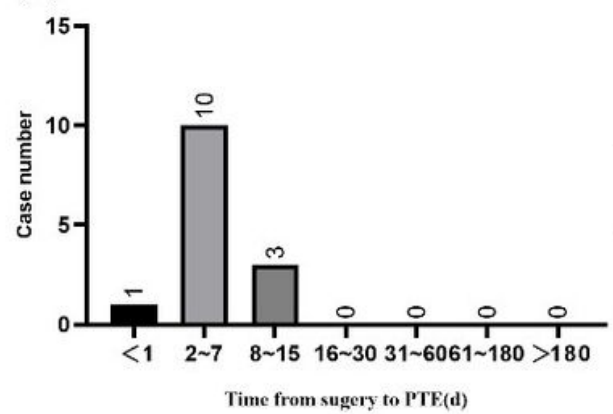

(b)

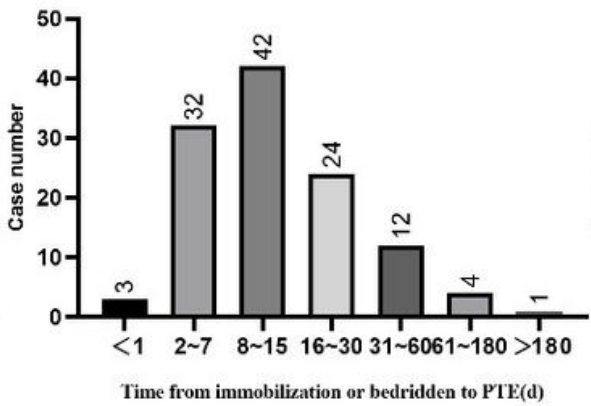

(e)

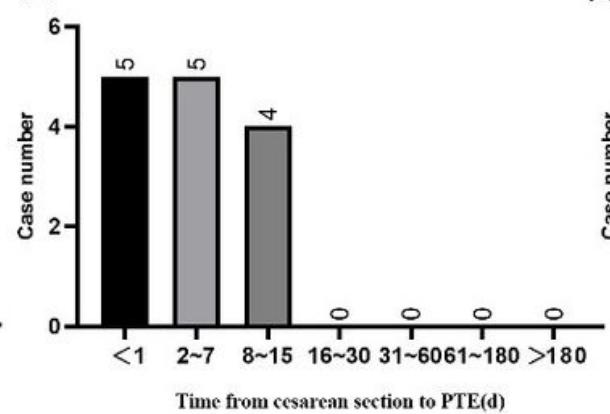

(c)

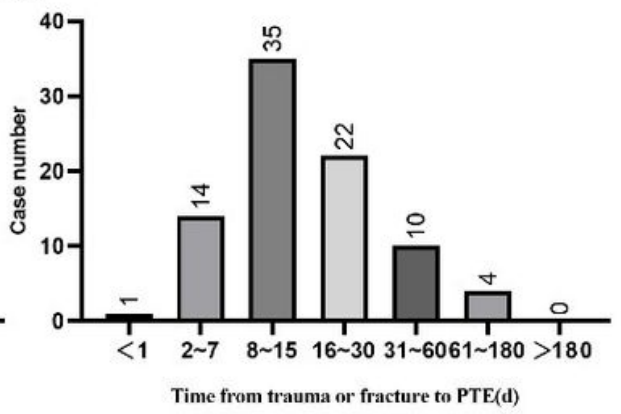

(f)

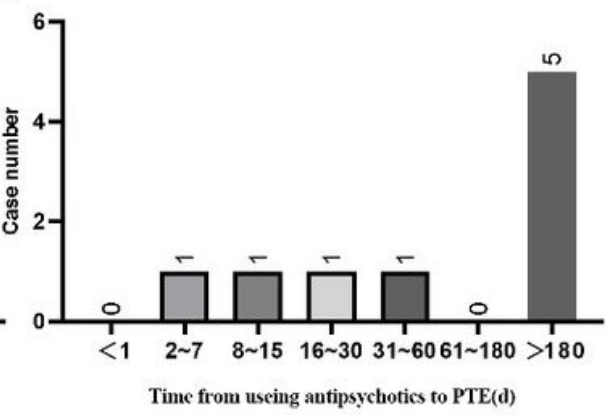

Figure 1

Distribution of the interval from the occurrence of risk factors to the PTE. (a) The time from the occurrence of the main risk factors to the PTE in all cases. $(b-f)$ The time from the occurrence of the top five risk factors to the PTE. 


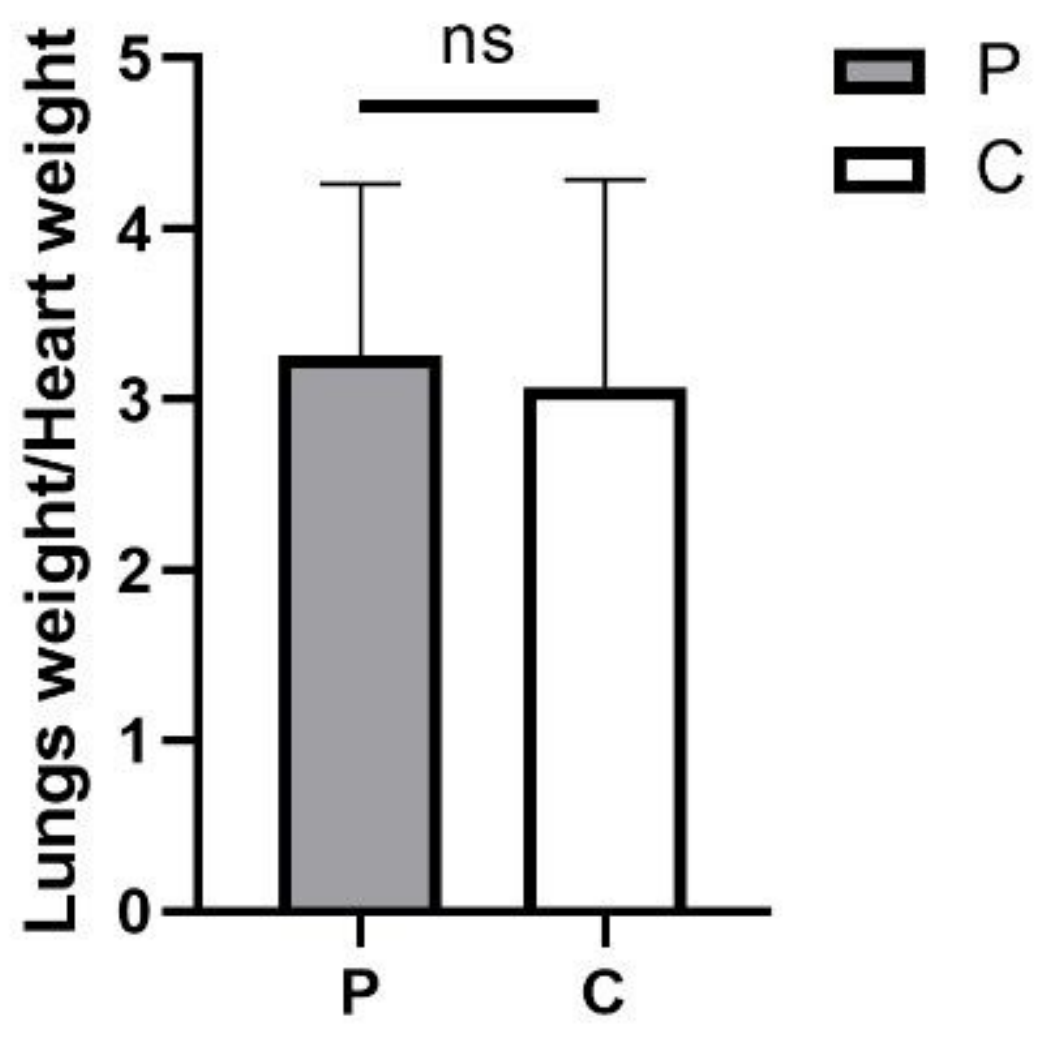

Figure 2

The lung-to-heart weight ratio in the PTE (P) and control (C) groups. No significant difference can be observed (Pख0.05). 